\title{
Transalpina
}

\section{Per un commercio di libri transalpino : l'inedito « diario di viaggio » di Giuseppe Molini a Parigi (10 giugno - 24 agosto 1844)}

\section{Marius Rusu}

\section{(2) OpenEdition}

\section{Journals}

\section{Edizione digitale}

URL: http://journals.openedition.org/transalpina/300

DOI: 10.4000/transalpina.300

ISSN: 2534-5184

\section{Editore}

Presses universitaires de Caen

\section{Edizione cartacea}

Data di pubblicazione: 1 octobre 2018

Paginazione: 85-100

ISBN: 978-2-84133-900-6

ISSN: 1278-334X

\section{Notizia bibliografica digitale}

Marius Rusu, «Per un commercio di libri transalpino : l'inedito « diario di viaggio » di Giuseppe Molini a Parigi (10 giugno - 24 agosto 1844) », Transalpina [Online], 21 | 2018, online dal 19 décembre 2019, consultato il 19 novembre 2020. URL : http://journals.openedition.org/transalpina/300 ; DOI : https:// doi.org/10.4000/transalpina.300 


\title{
PER UN COMMERCIO DI LIBRI TRANSALPINO : L'INEDITO «DIARIO DI VIAGGIO " DI GIUSEPPE MOLINI A PARIGI (10 giugno - 24 agosto 1844)
}

\begin{abstract}
Riassunto: Attraverso lo studio di un corpus organico di lettere conservate presso la Biblioteca Nazionale Centrale di Firenze, ricostruiamo il viaggio a Parigi, nel periodo giugno-agosto 1844, dell'editore fiorentino Giuseppe Molini. Le epistole che Giuseppe Molini invia al figlio Luigi, titolare di una delle imprese editorial-librarie più in vista del Granducato di Toscana e della stessa penisola, riportano osservazioni su una capitale francese vivace, sugli esuli italiani riparati, sull'editoria parigina e su un commercio librario transalpino fiorente. Il materiale documentario oggetto di questo articolo è un tassello inedito per illuminare un quadro culturale in fermento.

Résumé: À travers l'étude d'un corpus organique de lettres conservées à la Bibliothèque nationale centrale de Florence, on reconstruit le voyage à Paris, pendant les mois de juin à aô̂t 1844, de l'éditeur florentin Giuseppe Molini. Les lettres que Giuseppe Molini envoie à son fils Luigi, titulaire d'une des maisons d'édition et de librairie les plus en vue du Grand-Duché de Toscane et de la péninsule italienne, contiennent des observations sur la vie vivace de la capitale française, sur les exilés italiens qui s'y sont installés, sur l'édition parisienne et sur le commerce florissant des livres italiens. Les sources documentaires, objet de cet article, sont des pièces inédites qui éclairent un cadre culturel en effervescence.
\end{abstract}

Il 10 giugno 1844, Giuseppe Molini ${ }^{1}$, stampatore e libraio fiorentino erede di un'affermata famiglia di editori, si imbarca a Livorno per un viaggio che lo porterà a Parigi per più di due mesi. I motivi del viaggio sono duplici: commerciali, data la dimensione internazionale che caratterizzava l'impresa Molini, connessa a una rete del libro «europea» i cui esordi si fanno risalire alla seconda metà del Settecento ${ }^{2}$, e turistici; nel 1844 Parigi

1. G. Ajazzi, Operette bibliografiche del cavalier Giuseppe Molini, Firenze, Cellini, 1858.

2. Romualdo Molini era stato "procaccia» nel veneziano e, dopo essersi stabilito a Firenze nel 1757, aveva passato il testimone al figlio Giuseppe Molini senior; quest'ultimo grazie a contatti consanguinei a Londra e Parigi con i fratelli Pietro e Gian Claudio, aveva istituito un'autentica «multinazionale» del libro; si veda la voce corrispondente firmata da P. Scapecchi nel Dizionario Biografico degli Italiani, Roma, Istituto della Enciclopedia italiana, vol. 75, 2011. 
ospita l'Esposizione nazionale dei prodotti industriali, evento salutato come uno sforzo rinnovato di aspirare a un primato scientifico della Francia sul piano europeo ${ }^{3}$.

Le quindici lettere mai pubblicate che Giuseppe Molini invia a Firenze durante il soggiorno parigino, conservate presso la Biblioteca Nazionale Centrale di Firenze ${ }^{4}$, sono uno scambio organico con il figlio Luigi (chiamato affettuosamente "Gigi»), titolare della libreria di famiglia. Nelle epistole Giuseppe Molini non si limita a brevi resoconti intorno al viaggio e agli affari di cui tratta, ma offre uno sguardo originale e intimo sulle vicende che interessano la propria famiglia, sulla circolazione transalpina dei libri, sull'ecosistema editoriale parigino, su intellettuali di spicco (Giampietro Vieusseux e Jacob Gråberg di Hemsö, per citarne un paio), sulla vita nella capitale francese. Visto da questa angolazione il corpus di lettere avrebbe più il carattere di un «diario di viaggio ", espressione quanto mai pertinente ma che rischia di banalizzare il materiale. Non si tratta infatti di lettere unidirezionali riportanti l'esclusivo punto di vista di Giuseppe Molini, bensì di loci di dialogo una parte consistente delle epistole recano tracce dei commenti di Luigi Molini, che usa apporre a lato dei paragrafi commenti, osservazioni caustiche o semplici promemoria spuntati, a indicare l'effettiva chiusura di pratiche commissionategli dal genitore. Queste note a margine danno vita a un «dialogo » atipico, eppure rivelatore di uno scontro tra due generazioni di librai-stampatori, padre e figlio; gli argomenti che suscitano conflitto sono la gestione della libreria, i rapporti con clienti e fornitori, la politica degli sconti, le insinuazioni di Giuseppe Molini sulla cattiva gestione della ditta da parte di Luigi.

Uno studio puntuale su questo carteggio ha l'opportunità di portare a galla sguardi inediti su un periodo storico significativo, allargando il panorama culturale dell'epoca ed evidenziando lo stretto legame editoriale tra Italia e Francia alla vigilia dell'infuocata stagione del 1848.

\section{Parigi, faro editoriale}

La prima lettera che Giuseppe Molini invia al figlio è datata 10 giugno 1844 (431/12), nella quale condivide alcune informazioni di servizio e informa della partenza imminente da Livorno, insieme a due compagne di viaggio.

3. L'industrie: exposition des produits de l'industrie française en 1844, Parigi, L. Curmer, 1844, p. 2. Si veda anche G. Halphen, Rapport sur l'exposition publique des produits de l'industrie française de 1844, Parigi, Schneider et Langrand, 1845.

4. Segnature Mss. Vari 431/12-26, d'ora in poi abbreviate. Nella trascrizione epistolare, al posto di una riproduzione diplomatica, si è scelto di adattare alcuni elementi all'uso moderno, mantenendo le peculiarità e lo stile delle lettere. 
Il mare poco mosso agevola il viaggio per Marsiglia, città dalla quale il libraio fiorentino scrive il 12 giugno (431/13). Il proposito è partire per Lione il 13 giugno alle 7 di mattina, nonostante il rincaro delle diligenze dovuto all'Esposizione di Parigi. Oltre a un incontro con il quinto barone di Vernon sul «vapore» ${ }^{5}$, Giuseppe Molini lamenta di aver mancato per poco il cavaliere Simone Peruzzi, ministro residente in Belgio e Francia per il Granducato di Toscana ${ }^{6}$.

«Fracassati dal caldo, dalla polvere e dalle perdute notti», la comitiva giunge a Parigi alle 4 di mattina del 17 giugno (431/14), prendendo alloggio all'Hôtel Taitbout, situato al numero 10 della via omonima e poco lontano dai giardini del Palais-Royal. Insensibile alle fatiche del viaggio, il libraio fiorentino si reca subito alla nota libreria Galignani, con la quale intrattiene rapporti stretti, evidenziati nelle lettere successive ${ }^{7}$.

Da sabato 22 giugno (431/15) scompare il tono aneddotico e le lettere acquistano una professionalità gagliarda, tipica di una classe borghese in ascesa alla quale Giuseppe Molini appartiene a pieno titolo:

Sento con piacere che hai spedito la cassa n. 962. Dopo la partenza delle donne ho lavorato come un galeotto e spero di far partire stasera due belle casse che sono state di infinito dettaglio e conterranno quasi tutti articoli di pronto realizzamento. [...] Così sarà affatto inutile che io aspetti l'invio che farà Galignani con la diligenza il dì primo di luglio per mandarci quei libri che mi chiedi col detto mezzo. Anzi manderò in queste casse anche un nuovo romanzo di Israeli pubblicato da Baudry ${ }^{8}$ (431/15).

5. Dictionary of National Biography, S. Lee (a cura di), Londra, Smith, Elder, vol. 58 (Ubaldini - Wakefield), 1899, p. 275.

6. Almanacco Toscano, Firenze, Stamperia Granducale, 1844, p. 171.

7. Giovanni Antonio Galignani aprì il suo negozio di libri inglesi e stranieri nel 180o, impresa continuata dai figli John Anthony e William dopo la sua morte nel 1821 . Si veda G. Barber, Galignani's and the Publication of English Books in France from 1800 to 1852, Londra, The Bibliographical Society, 1961; D. Cooper-Richet ed E. Borgeaud, Galignani, Parigi, Galignani, 1999.

8. Il romanzo di Benjamin Disraeli, politico d'oltremanica e futuro primo ministro del Regno Unito, è Coningsby or the New Generation, pubblicato a Londra nel 1844 da Henry Colburn e ristampato a Parigi lo stesso anno da Baudry. Sul romanzo si faccia riferimento a Le Semeur, journal philosophique et littéraire, Parigi, Au bureau du Semeur, 1844, t. XIII ( du $1^{\text {er }}$ janvier au 31 décembre), p. 276. L'editore Baudry, controverso e spesso accusato di "pirateria ", "dès les années $1840,[\ldots]$ a développé une stratégie commerciale en direction des écoles, des lycées et des universités" (in Les mutations du livre et de l'édition dans le monde du XVIII siècle à l'an 2000 (Actes du colloque international Sherbrooke 2000), J. Michon e J.-Y. Mollier (a cura di), Laval (Canada) - Parigi, Presses de l'Université Laval - L'Harmattan, 2002, p. 135); su Baudry anche D. Cooper-Richet, «L'imprimé en langues ètrangéres à Paris au XIX ${ }^{e}$ siècle: lecteurs, éditeurs et supports", Revue française d'histoire du livre, $\mathrm{n}^{\circ}$ 116-117 (Nouvelle série), p. 213-235; J.-B. Francou, Baudry, un éditeur 
Argomenti della lettera sono anche una spedizione dei Misteri di Parigi di Eugène Sue e un reclamo all'editore Dubochet intorno all'Illustration, di cui Molini si fa portavoce a nome di un cliente fiorentino ${ }^{9}$.

Una settimana dopo, l'editore fiorentino scrive al figlio da un nuovo indirizzo (lettera del 29 giugno, 431/16), l'hôtel Valois in rue de Richelieu, 71, dove Giuseppe Molini aveva già alloggiato, definendolo «mio antico albergo ${ }^{10}$. Emerge la pena per le due compagne di viaggio, che avevano proseguito il viaggio verso Londra, della cui sorte il nostro scrive al «Cugino ». Si tratta di Charles Frederick Molini, figlio di Pietro Molini del ramo inglese della famiglia e libraio al n. 17 di Paternoster Row, fornitore della Royal Academy e della biblioteca del British Museum ${ }^{11}$. Dopo aver constatato l'arrivo a Marsiglia della cassa n. 962, Giuseppe Molini si incarica inoltre di una commissione per Duprat, libraio parigino legato all'Institut de France, alle Società asiatiche di Parigi e Londra e alla Biblioteca Reale, fornendo una lista al figlio Luigi: volumi di Dante, il Bullettino delle leggi di Toscana, ma soprattutto Lo specchio di Marocco di Gråberg ${ }^{12}$, opera ritenuta capace di catalizzare interesse data la situazione politica, che vedeva la Francia in procinto di annettere l'Algeria ai suoi con la forza delle armi ${ }^{13}$. Fa capolino, in calce alla lettera, un Molini intermediario di opere "pericolose»; raccomanda al figlio Luigi di avvisare "Lemonnier che Brockhaus non ha mai ricevuti gli ultimi 50 Arnaldo, che è qui molto domandato dagl'Italiani ${ }^{14}$. Non stupisce l'interesse degli esuli a Parigi per libri all'indice, e neppure lo smercio che ne fa Brockhaus, titolare di

pirate du XIX eiècle, ou l'histoire de la Librairie européenne de 1815 à 1852, mémoire de maîtrise dirigé par D. Cooper-Richet et J.-Y. Mollier, Université de Versailles SaintQuentin-en-Yvelines, 1999; M.I. Palazzolo, «Un editore francese in lingua italiana : Louis Claude Baudry", in I tre occhi dell'editore. Saggi di storia dell'editoria, Roma, Archivio Guido Izzi, 1990, p. 23.

9. «Roderigo Macleod gentiluomo inglese abitante in Firenze a Palazzo Vernaccia in Borgo Pinti ", Gazzetta di Firenze, n 94, martedì 6 agosto 1844, p. 4.

10. Dalla stessa lettera: "Io sgombererò domani, così risparmierò franchi 10 al mese e mi avvicinerò a tutti i miei affari (eccettuati i soli Truchy, Delay e il ministro di Toscana, del quale ultimo non mi importa un fico) [...] e avrò una camera un po' meno elegante, ma assai più grande e con più comodi. In quella ove sono ora tocco il palco con le mani sebbene salgo 79 scalini ».

11. P.R. Harris, A History of the British Museum Library (1753-1973), Londra, The British Library, 1998, p. 143.

12. Stampato a Genova nel 1834 con i tipi di Pellas.

13. Sul conflitto in Algeria cf. P. Montagnon, La conquête de l'Algérie, Parigi, Pygmalion, 1986, e B. Stora, Histoire de l'Algérie coloniale (1830-1954), Parigi, La Découverte, 2004.

14. È il libro contro la tirannia di G.B. Niccolini, Arnaldo da Brescia, stampato in clandestinità a Marsiglia e introdotto a Firenze con un trucco doganale, si veda C. Ceccuti, Un editore del Risorgimento. Felice Le Monnier, Firenze, Le Monnier, 1974. 
una libreria poco distante dall'alloggio di Giuseppe Molini ${ }^{15}$. Allegata all'epistola, una fattura corposa elenca nel dettaglio il contenuto di « due grosse casse marcate LM [Luigi Molini] n. 103.104, imballate e partite da Parigi col roulage acceléré il dì 24 giugno 1844 [...]. Dovranno essere a Marsiglia in dodici giorni ». Le casse contengono anche articoli e fatture per la Biblioteca Palatina di Firenze e tracce di un conto con i librai editori Treuttel \& Würtz, risalente almeno al $1838^{16}$. L'elemento che più spicca è una raccomandazione importante di Giuseppe Molini, tale da essere marcata con lo schizzo di una manicula:

Alla cassa 103 ho fatto contrassegnare il coperchio con due oo. Si operi bene in dogana di farla aprire da questa parte perché nel fondo ci sono copie 13 Destini d'Italia distese e accomodate in modo che potranno facilmente nascondersi. Sopra esse vi sono dei fogli e sopra questi i fascicoli grandi (431/16).

L'opera citata che si tenta di contrabbandare nel Granducato, a dispetto del titolo, è Le speranze d'Italia di Cesare Balbo, come conferma la lettera datata 4 agosto (431/23). Il libro era uscito lo stesso anno a Parigi, fomentando un dibattito che fluttuava "tra l'impostazione ideologica del "Primato" e quella pragmatica delle "Speranze" ${ }^{17}$. Le implicazioni sono duplici, e oscillano tra la volontà di concludere un affare redditizio, perché tale da meritare un'azione clandestina, e l'intento di affrontare i rischi in fase doganale per un testo richiestissimo ${ }^{18}$. Siamo in un anno, il 1844, terreno di idee diverse del nazionalismo italiano che portano alla luce un'autentica «questione sociale», destinata ad acuirsi nel triennio successivo ${ }^{19}$.

15. "À partir du 7 courant, la librairie allemande, française et étrangère de Brockhaus et Avenarius sera entièrement transférée rue de Richelieu, $n .69$, au ${ }^{e r}$, vis-à-vis la Bibliothèque royale" (Feuilleton du Journal de la librairie, $\mathrm{n}^{\circ} 27$, samedi 2 juillet 1842).

16. A. Hass, «Un libraire fournisseur de grandes bibliothèques européennes: Treuttel \& Würtz», in Strasbourg, le livre et l'Europe, XV $V^{e}-X X I^{e}$ siècle, Y. Sordet e F. Barbier (a cura di), Genève, Droz, 2016.

17. A. Scirocco, L'Italia del Risorgimento, Bologna, Il Mulino, 1997, p. 227.

18. L'opera di Balbo venderà 80000 copie tra il 1843 e il 1848. "Esprimere un giudizio sulla censura toscana non è agevole, tuttavia quello di una minore severità rispetto a quelle degli altri Stati preunitari espresso da altri studiosi sembra essere condivisibile, la maggiore facilità nel ricevere giornali e periodici dall'estero e la possibilità di importare liberamente anche quei testi respinti dalla censura, la cui introduzione non fosse stata esplicitamente vietata, faceva sì che i toscani potessero aggirare legalmente i divieti censori" (D.M. Bruni, Potere e circolazione delle idee. Stampa, accademie e censura nel Risorgimento italiano, Milano, Franco Angeli, 2007, p. 57, 340).

19. F. Della Peruta, L'Italia del Risorgimento. Problemi, momenti e figure, Milano, Franco Angeli, 1997, p. 168. 
Gli anni Quaranta, per gli esuli italiani, rappresentano l'opportunità di una « costruzione deliberata, consapevole della nazione unitaria attraverso la cultura e la lingua $»^{20}$. Non occorre annoverare i Molini nella schiera di quegli editori «militanti ${ }^{21}$, poiché mancano indizi su tendenze risorgimentali nella famiglia e siamo a conoscenza dello stretto legame con la nobiltà del Granducato; a questi elementi vanno aggiunti i riguardi di Leopoldo II di Lorena nei confronti di Giuseppe Molini, meglio esplicitati nella lettera del 14 luglio (431/19). Resta il punto centrale, ovvero il coinvolgimento diretto dei Molini, padre e figlio, nell'immissione di libri «perniciosi» in una realtà, quella toscana, ricettiva a idee di unità nazionale, dove le maglie della censura erano lasciate larghe e neppure un rescritto sovrano (promulgato il 10 marzo 1837) aveva inasprito il controllo ${ }^{22}$.

La lettera del 5 luglio (431/17) esordisce con un'aspra invettiva nei confronti di un «bibliotecario di Pistoia», reo di un gesto sgradevole per aver affidato ai Molini e a Vieusseux il reperimento di una stessa opera; il trucco è svelato quando Giampietro Vieusseux si rivolge proprio ai Molini per il recupero del volume. Emerge un sentimento intollerante verso il libraio franco-svizzero trapiantato a Firenze, con parole accese di Giuseppe Molini nei confronti del Vieusseux nella suddetta lettera, "tanto più che quest'ultimo mancante di mezzi, come è naturale non avrebbe trovato mai $^{23}$ quel tomo [...]. Bisogna far sentire al signor bibliotecario quanto sia disdicevole il ricorrere a uno straniero incapace piuttosto che a un abile concittadino!». Ricaviamo il titolo dell'opera contesa da un appunto in inchiostro rosso di Luigi Molini, che annota "Poliglotta di Walton» a lato del paragrafo; è la Bibbia curata da Brian Walton, seicentina di valore $^{24}$. La questione è messa in disparte a fronte di un dispiacere per il libraio fiorentino, che confessa avere appreso della morte della figlia di un collega parigino:

20. S. Tatti, «Esuli e letterati: per una storia culturale dell'esilio risorgimentale», in L'officina letteraria e culturale dell'età mazziniana (1815-1870), Q. Marini, G. Sertoli e S. Verdino (a cura di), Novi Ligure, Città del silenzio, 2013, p. 89.

21. Come poteva essere stato Ruggia di Lugano, G. Martinola, Un editore luganese del Risorgimento: Giuseppe Ruggia, Lugano, Fondazione Ticino Nostro, 1985; F. Mena, Stamperie ai margini d'Italia. Editori e librai nella Svizzera italiana 1746-1848, Bellinzona, Edizioni Casagrande, 2003.

22. Archivio di Stato di Firenze, Segreteria di Stato 1814-1849, B. 527, prot. 36, $\mathrm{n}^{\circ} 26$.

23. Parola sottolineata.

24. Stampata a Londra nel 1657 presso Thomas Roycroft. Il Catalogo dei libri che si trovano attualmente vendibili presso Molini, Landi, e Co., compilato dallo stesso Giuseppe Molini, lo valuta 1200 paoli fiorentini nel 1807, p. 8 . 
Un'altra morte mi ha fatto molto colpo. Il povero Baudry ha perduto poco tempo fa la sua figliola abilissima, che aveva maritata a un giovine il quale gli era succeduto nell'antica sua bottega Rue du Coq. Erasi maritata da pochi anni ed era in Parigi quando si maritò ed ero stato anche invitato, sarà 4 anni, al ballo di nozze. Pare sia morta di febbre puerperale come la povera Incontri ${ }^{25}(431 / 17)$.

Una fattura allegata rafforza l'idea di una stretta relazione tra i Molini di Firenze e Galignani di Parigi; riporta la spedizione di un «ballotto » a nome Galignani, mezzo diligenza, in data $\mathrm{I}^{\circ}$ luglio. Giuseppe Molini approfitta per accludervi alcuni articoli destinati al figlio Luigi e commenta, a lato, la scontistica sui pacchetti per fare acqua di seltz e l'uscita sul Constitutionnel dell'Ebreo errante di Sue: "Questo è un nuovo scritto di Sue che fa gran chiasso [...]. Esso viene pubblicato regolarmente a pezzi nel fogliettone del giornale». La lettera si conclude con alcuni paragrafi aneddotici rivolti alla figlia Fanny Molini e alla nipote Giovanna in cui esalta Parigi come un «paradiso", afferma di voler estendere il soggiorno quanto possibile salvo richiami in Toscana di Neri Corsini o Giuseppe Paver ${ }^{26}$, ed esalta l'Esposizione nazionale dei prodotti industriali, « una cosa impossibile a descriversi, e da rimanere incantati ». L'infatuazione di Giuseppe Molini è difficile a criticarsi. Parigi nel 1844 è un calderone di contraddizioni, una città che trasuda progresso e ottimismo ${ }^{27}$, eppure considerata al contempo nucleo di una Francia «dove non si può pensare» perché «tutti i buoni pensieri qui non si possono dire $»^{28}$.

Le pratiche doganali sono il tema centrale dell'epistola datata 13 luglio (431/18), questa volta dalla prospettiva francese, dove Giuseppe Molini ammonisce il figlio Luigi sulle future spedizioni:

25. La Incontri citata è la marchesa Ortensia consorte di Attilio Incontri, secondogenita di Gino Capponi morta nel maggio 1844: Epistolario di Giuseppe Giusti, F. Martini (a cura di), Firenze, Le Monnier, 1932, p. 56; Carteggio Tommaseo - Vieusseux, V. Missori (a cura di), Firenze, Le Monnier, 2002, p. 246.

26. Rispettivamente primo ministro e ministro dell'interno del Granducato di Toscana.

27. A. Monti, Un italiano: Francesco Restelli (1814-189o), Milano, Società nazionale per la storia del Risorgimento italiano, Comitato regionale lombardo, 1933, p. 13. «Le ragioni particolari del fascino di Parigi vanno cercati nella società parigina dell'Ottocento: una società brillante, spregiudicata, incredula, cinica, che si divideva ordinatamente in "bel mondo", borghesia ricca e borghesia minore, per sfociare poi nella gran marea del popolo e della plebe; una società organizzata, capace di esercitare un'attrattiva costante sul forestiero » (I. Taine, Appunti su Parigi vita ed opinioni di Tommaso Graindorge, G.B. Angioletti (a cura di), Milano, Editoriale Domus, 1945, p. 7).

28. L. Palmieri, «Come si vive a Parigi», La voce della verità, vol. 9, ${ }^{\circ}$ 1369, 1840, p. 1208. 


\begin{abstract}
Prima di tutto all'avvenire bisognerà che tu faccia muso duro e ricusi assolutamente di ricevere qualunque pacchetto serrato che ci sia rimesso per mandarsi qua. Se vi saranno libri stampati in Francia e più che altro libri francesi stampati in Italia, e qualunque altro oggetto che non sia libri bisogna ricusare di riceverli. Gli imbarazzi di questa dogana sono incredibili. Sono dieci giorni che la cassa n. 962 è arrivata qui con l'acceléré e non si è potuta ancora avere. La maggior difficoltà si è incontrata per quel maledettissimo pacchetto consegnato da quel malanno di Millingen ${ }^{29}$ con il quale bisognerà romperla. Le gite che ho dovuto fare per questo sono incredibili e sono costate spesa, oltre al perdimento di tempo e di danno incalcolabile di aver dovuto ritardare per tanti giorni il ricevimento della cassa (431/18).
\end{abstract}

La questione non è secondaria, trattandosi di una transazione commerciale che impegna a fondo le risorse degli editori. Il blocco in dogana si traduce in una crescente esposizione finanziaria e in perdite economiche; la congiuntura temporale è rilevante, perché il 1844 è il penultimo anno di un periodo protezionistico tipico della monarchia di Luglio, che prelude a una progressiva liberalizzazione fino alla Terza repubblica ${ }^{30}$. La nota seguente, che interessa un debito verso gli asili infantili di Firenze, traccia un profilo dei Molini membri della Società per gli Asili Infantili di Firenze, alla quale risultano come sottoscrittori sia il padre Giuseppe, sia i figli Luigi e Fanny ${ }^{31}$. Il doppio binario che lega i mondi editoriali francese e italiano è messo in evidenza quando nella lettera si arriva al conflitto franco-algerino. Sull'Algeria Cesare Airoldi ${ }^{32}$ ha espresso interesse e comunicato a Luigi Molini la commessa di altri volumi sulla questione:

Al cavalier Airoldi dirai che finora sull'Algeria non è uscito altro di buono che l'opera di cui abbiamo vendute più copie avute da Furne ${ }^{33}$ (e della quale conviene dirmi se ne ho da provvedere altre). Finora quelli che

29. James Millingen, archeologo inglese di fama, residente a Firenze all'epoca e autore di due opere sulla numismatica stampate con il marchio dei Molini.

30. F. Barbier, «Les marchés étrangers de la librairie française», in Histoire de l'édition française, R. Chartier, H.-J. Martin (a cura di), Parigi, Fayard - Cercle de la Librairie, vol. 3, Le temps des éditeurs. Du romantisme à la Belle Époque, 1990, p. 309.

31. Quest'ultima coinvolta in prima persona: la figlia Giovanna, nipote di Giuseppe Molini, frequenta l'Istituto privato diretto da E. Siry in via dello Studio $\mathrm{n}^{\circ} 765$; si veda il Settimo rapporto sugli asili infantili di Firenze, Firenze, Tipografia della Speranza, 1841, p. 67 e 75 , e la sua edizione ottava, Firenze, Nella Stamperia Granducale, 1845, p. 109.

32. Esule palermitano stabilitosi a Firenze nel 1824 e facente parte della cerchia intellettuale che ruotava attorno al marchese Gino Capponi e a Giampietro Vieusseux.

33. È il libraio parigino Charles Furne, E. Rosseeuw Saint-Hilaire, Notice sur Charles Furne, Parigi, Imprimerie J. Claye, 1860. L'opera citata è L. Galibert, L'Algérie ancienne et moderne, Parigi, Furne et $C^{\text {ie }}, 1844$. 
hanno scritto su quel soggetto sono militari. Si crede però che sarà fatta in seguito qualche altra opera più significante. Ora il possesso è troppo fatto di fresco $(431 / 18)$.

Il tono dell'epistola è grave quando si accenna alla morte di Giuseppe Menotti ${ }^{34}$, padre del patriota Ciro impiccato a Modena tredici anni prima, residente a Batignolles, comune adiacente Parigi ${ }^{35}$; nonché alla morte del «ricchissimo libraio» Panckoucke, evento che Giuseppe Molini non crede comporterà la sospensione della pubblicazione della sua collezione di classici latini. Nella chiusa della lettera il libraio fiorentino si augura che il figlio Luigi abbia già ricevuto le casse n. 104 e 105 (partite da Parigi il 24 giugno) e che numerose delle commissioni al loro interno siano già state eseguite. A conferma di un legame che sembra andare oltre le asettiche transazioni d'affari, i saluti finali a Luigi Molini sono affidati anche alla penna dell'editore-libraio parigino Duprat ${ }^{36}$, oltre a quella del padre. I legami che Molini intratteneva nella capitale francese erano basilari per la rete editoriale che connetteva Francia e Italia, e di riflesso l'Europa. Le note di carico delle casse che Giuseppe Molini spedisce da Parigi evidenziano un commercio multiforme, fatto di libri antichi, giornali e novità come i romanzi di Sue e Disraeli. Traspare la volontà di instaurare una simbiosi con i librai e editori parigini, in un rapporto di cooperazione che assicuri benefici reciproci. Nelle lettere di Giuseppe Molini non sono presenti, infatti, elementi che portino a sospettare ostilità commerciali con le gens du livre della capitale francese, abbondano invece spunti positivi e una ricerca spasmodica di opportunità editoriali.

\section{Thiers e il Progetto di riordinamento delle biblioteche fiorentine}

Puoi figurarti quanto rimasi sorpreso tornando a casa ieri sera nel trovare un dispaccio di Sua Eccellenza il nostro ministro dell'interno signor Paver ${ }^{37}$ con la

34. «Avrai sentite le nuove del povero vecchio Menotti. Ebbi anch'io l'invito di andare a' suoi funerali, ma egli abitava fuori di Parigi, e non vi andai».

35. T. Grandi, Ciro Menotti e i suoi compagni o le vicende politiche del 1821 e 1831 in Modena, Bologna, Tipografia della Società Azzoguidi, 188o, p. 350. Giuseppe Menotti fu commerciante a Carpi con contatti transalpini, cf. A.G. Spinelli, Memorie sull'arte del truciolo in Carpi, Modena, Tipo-litografia di Luigi Rossi, 1905, p. 301.

36. Si veda la lettera del 29 giugno, $431 / 16$.

37. "Uomo mediocre il cui principale merito era quello di essere stato a suo tempo il segretario di Ferdinando III, e di avere fama di "austriacante" di stretta osservanza» (G. Ciappelli, Un ministro del Granducato di Toscana nell'età della Restaurazione. Aurelio Puccini e le sue "Memorie», Roma, Edizioni di Storia e Letteratura, 2007, p. 42). Riferimenti anche in G. Baldasseroni, Leopoldo II Granduca di Toscana e i suoi tempi, Firenze, Tipografia all'Insegna di S. Antonio, 1871, p. 159. 
quale, trattandomi da illustrissimo, mi partecipa che Sua Altezza Imperiale e Reale gli ordina di farmi sapere essere sua intenzione di valersi delle mie cognizioni e della mia esperienza per cooperare alla riordinazione delle biblioteche di Firenze. Che in conseguenza Sua Altezza Imperiale e Reale risolvè il 4 corrente di creare una commissione a questo oggetto, della quale ci dovrò far parte, $\mathrm{e}$ di parteciparmi questa notizia avvisandomi che ogni altra partecipazione resti sospesa fino al mio ritorno in Firenze. Questa partecipazione è accompagnata da un gentile biglietto confidenziale ed autografo del Paver con la quale mi invita a fargli sapere qualche cosa sull'epoca del mio ritorno, della quale cosa egli mi prega ancora nella comunicazione officiale (431/19).

Accreditato di un onore che difficilmente altri bibliotecari o editori-librai avrebbero disdegnato, Giuseppe Molini comunica al figlio dell'incarico che gli si prospetta nella lettera del 14 luglio (431/19). L'esperienza del nostro si era consolidata circa un ventennio prima, quando era stato nominato prima bibliotecario aggiunto e poi effettivo alla Palatina di Firenze. Eppure le ombre intorno alla futura nomina di commissionario per il riordinamento delle biblioteche fiorentine sembrano sussistere, se Giuseppe Molini raccomanda al figlio discrezione nel divulgare la notizia, perché «l'offerta è bella e mi fa molto onore, ma sarà sorgente di molti dispiaceri, e preveggo che tutti codesti Bibliotecarii che ora sono tutti amici miei diventeranno miei accerimi nemici ${ }^{38}$. Al di là degli scarsi risultati che questa iniziativa otterrà, permane la questione di una necessità di riflessione intorno all'organizzazione e razionalizzazione di un'istituzione come quella bibliotecaria. Riflessione che Giuseppe Molini perseguirà con tenacia, dando alle stampe un breve opuscolo, al quale affida la comunicazione della sua proposta di riordinamento ${ }^{39}$. A lato della proposta granducale, che assorbe gran parte dell'attenzione in questa lettera del 14 luglio, vi sono le vicende della cassa n. 962, per le cui operazioni di scarico e fatturazione Molini padre prevede un tempo di almeno due giorni.

Da lettera datata 20 luglio (431/20) si apprende che Giuseppe Molini ha risposto al ministro Paver il 16 luglio, sia con una comunicazione ufficiale sia con una confidenziale, e ora attenderà notizie in merito. Non manca di condividere con il figlio Luigi la segnalazione sul libraio padovano

38. Sul progetto di riordinamento delle biblioteche fiorentine promosso da Leopoldo II si consulti D. Fava, "Un progetto di riforma delle biblioteche pubbliche di Firenze ", in Accademie e Biblioteche d'Italia, vol. 9, 1935, p. 475; M. Rossi, Bibliofilia, bibliografia e biblioteconomia alla corte dei Granduchi di Toscana Ferdinando III e Leopoldo II, Manziana, Vecchiarelli, 1996; P. Innocenti, Il bosco e gli alberi, Firenze, La Nuova Italia - Giunta regionale toscana, vol. 1, 1984.

39. G. Molini, Progetto di riordinamento delle pubbliche biblioteche di Firenze, Firenze, Tipografia di Giovanni Benelli, 1848. 
Luigi Rusconi: "Avrai saputo che il povero Rusconi libraio a Padova è stato mandato via per avere venduto dei libri proibiti, e ha sofferto gravi inquietudini ed è andato dal fratello a Bologna ${ }^{40}$.

Torna, nella lettera successiva del 27 luglio (giorno di commemorazione della rivoluzione del 1830 in cui anche Giuseppe Molini si reca a NotreDame a «suffragare» i morti, 431/21), l'attualità sul conflitto algerino, con un'esortazione al figlio per convincere Jacob Gråberg di Hemsö ${ }^{41} \mathrm{a}$ ristampare la sua opera Lo specchio di Marocco, ché « tutti lo domandano». Lo stesso Gråberg, in apparenza, sfrutta il ruolo alla Biblioteca Palatina per ordinare volumi che intende trattenere per sé; è il caso del Tableau synoptique représentant les noms, les émigrations, les filiations, l'origine, les caractères physiques et moraux des races de l'Afrique septentrionale, che Gråberg confonde come libro quando invece trattasi di un tableau di difficile reperimento ${ }^{42}$. Spicca più di ogni altra comunicazione la sezione della lettera intitolata "Progetto colossale», e la definizione è quanto mai pertinente. Trattasi di un progetto articolato che coinvolge Adolphe Thiers, deputato e figura politica centrale nella Parigi del $1844^{43}$, che Molini padre sottopone al figlio Luigi:

Ho parlato con monsieur Paulin ${ }^{44}$ il quale ha acquistato da monsieur Thiers il manoscritto della sua Storia del Consolato e dell'Impero. L'opera sarà in 10 vol. $8^{\circ}$ di 30 fogli circa. Ne uscirà un tomo al mese. Comincerà a stampare in settembre e si pubblicherà il primo volume a novembre prossimo. Il signor Paulin appena che avrà ricevuto ciascheduno foglio colla firma ed il bon à tirer del signor Thiers ne farà subito girare una copia e questa la manderà sotto fascia a Firenze per farne fare la traduzione. Per i tomi 1, 2, 3, il signor Paulin ci obbliga di non pubblicarli se non un mese dopo che avrà mandato l'ultimo foglio di chiaschedun tomo. I tomi 4 e seguenti ci obbliga di pubblicarli dopo 15 giorni come sopra, dovendo a quell'epoca l'operazione essere assicurata (431/21).

40. Su Luigi Rusconi si veda M. Callegari, Stampatori e librai a Padova nella prima metà dell'Ottocento, Saonara, Il Prato, 2013.

41. Intellettuale svedese trapiantato a Firenze, reggente alla Biblioteca Palatina e assiduo frequentatore del Gabinetto Vieusseux. Cf. F. Parlatore, "Elogio di Jacob Gråberg de Hemsö ", in Atti dell'Accademia dei Georgofili, XXVI, 1848, p. 290.

42. Bibliographie de la France, ou Journal général de l’imprimerie et de la librairie, vol. 33, n 890, samedi 17 février 1844 .

43. Un giudizio poco lusinghiero di Thiers è firmato da A. de Tocqueville, che lo dipinge capace di «addormentare la nazione e di rassicurare il re, in modo di insinuarsi fino al potere» (Vita attraverso le lettere, N. Matteucci e M. Dall'Aglio (a cura di), Bologna, Il Mulino, 1996, p. 82).

44. Jean-Baptiste-Alexandre Paulin, giornalista, editore e libraio parigino. Cf. F.E. Comparato, Books for the Millions. A History of the Men Whose Methods and Machines Packaged the Printed Word, Harrisburg (Pennsylvania), Stackpole, 1971, p. 92. 
L'offerta è accompagnata da una richiesta di 10000 franchi, ovvero 33,33 franchi a fascicolo, secondo i calcoli del nostro. Due editori di Torino hanno già espresso interesse e accettato le condizioni economiche, tuttavia Paulin, non conoscendo alcuno di loro, temporeggia in attesa di una risposta di Giuseppe Molini. Si accompagnano pensieri su edizioni contraffatte dell'opera e un pregiudizio:

È noto che una contraffazione non potrà farsi in Italia. Potrà qualcheduno fare una traduzione diversa, ma con le precauzioni suddette sarà difficile che qualcheduno ci pensi, perché noi avremo sempre un paio di mesi di avvantaggiamento di tempo. [...] Converrebbe fare, se l'affare conviene, trovare un socio danaroso che contribuisse. Qualche signore costì forse lo farebbe. In tal caso bisogna segnarsi bene e non ne fare nulla se il socio non è veramente galantuomo e soprattutto non ebreo (431/21).

Stimata la tiratura in 2 o 3000 copie, cifra dettata dalla "privativa in Italia fuorché a Napoli », Giuseppe Molini invita il figlio a esaminare a fondo la questione, soprattutto per il rapporto privilegiato che il libraio fiorentino vuole vantare con Paulin:

Avendo la sicurezza di non poter esser ristampati è certo che gli altri librai d'Italia daranno commissione di centinaia di copie. Con tutto ciò non ardisco consigliarlo né ricusarlo, e sta a te rifletterci con tranquillità domenica. [...] Secondo un calcolo fatto da Duprat ogni volume tutto compreso ci verrebbe a costare franchi 2.30 circa, tirando 3.000 copie $(431 / 21)$.

Dal tono assunto nella proposta si comprende meglio il ruolo di Giuseppe Molini nell'impresa di famiglia. Il nostro all'epoca ha settandue anni, un'età che gli consente ancora di dare il suo contributo (essenziale) al marchio editoriale cui appartiene, allo stesso modo di mantenere una posizione defilata dal punto di vista decisionale. Pur traghettata dall'esperienza di Giuseppe Molini, la società di famiglia è retta da Luigi Molini. Eppure negli anni Trenta la ditta Molini aveva rischiato il tracollo, causa la cattiva gestione di Luigi, tanto che le differenze di visioni tra padre e figlio sul mondo editoriale (e sulla maniera di condurre affari) si faranno strada prepotenti nelle ultime lettere di questo carteggio ${ }^{45}$.

45. Editori italiani dell'Ottocento. Repertorio, A. Gigli-Marchetti, M. Infelise e L. Mascilli Migliorini (a cura di), Milano, Franco Angeli, 2004. 


\section{Di esuli e libri proibiti}

Spigolando nelle due lettere del 31 luglio e 4 agosto (431/22-23) si scopre che l'affare Thiers non va in porto, ad assicurarsi la traduzione è l'editore Vaccarino, il primo a scrivere a Paulin ${ }^{46}$. Le attenzioni si concentrano su questioni diverse, come il sequestro in dogana toscana delle Speranze d'Italia di Cesare Balbo e delle opere del filosofo francese Félicité de Lamennais, opere spedite il 24 giugno nella cassa n. 103. A conferma di una noncuranza diffusa per le questioni doganali nel Granducato, Giuseppe Molini non appare preoccupato dal sequestro, e raccomanda al figlio di lasciare in giacenza i libri in dogana e attendere il suo ritorno, tanto più che le Speranze d'Italia « tutti dicono essere un gran libraccio ${ }^{47}$. Lo scarso cruccio del libraio fiorentino è spia di un sistema consolidato che permetteva agli editori toscani di eludere la sorveglianza e la censura ${ }^{48}$. Anche la cronaca di Parigi ha un ruolo nell'epistola, con Giuseppe Molini che confessa avere assistito ai giochi pirotecnici e di illuminazione in Place de la Concorde e sugli Champs-Élysées, schivando la tragedia che i giornali riportano il 30 e il 31 luglio: a causa della ressa e della confusione si erano creati disordini che avevano portato alla morte di tre persone e al ferimento di nove ${ }^{49}$ :

Tutti i giornali di Parigi non parlano che delle disgrazie accadute lunedì sera sulla Piazza della Concordia e ai Campi Elisi. Voglio che sappiate dunque che andai anche io alla festa ma che prudentemente scansai la folla, vidi benissimo ogni cosa e alle 11 me ne stavo tranquillamente a letto. Ė impossibile descrivere l'effetto magico di quella meravigliosa illuminazione che per un viale larghissimo cominciava dal palazzo delle Tuileries e finiva all'Arco della Stella, cioè per lo spazio di almeno due miglia in linea retta. Superava la

46. «Firenze dì 28 agosto 1844. I sottoscrittori editori della Storia del Consolato e dell'Impero di Napoleone scritta in francese dal signor Thiers, prima edizione in lingua italiana, avendo concesso l'esclusiva per tutta la Toscana al signor Lelio Arbib di Firenze, invitano i loro corrispondenti a rivolgersi al medesimo per le commissioni che ne volessero dare. NB. In forze del contratto da noi stipulato con gli editori di Parigi ci viene garantito $1^{\circ}$ il diritto esclusivo delle edizioni in lingua italiana; $2^{\circ} l^{\prime}$ invio per la posta dei fogli si stampa mano mano che si andranno ponendo sotto i torchi in Parigi. Per la qual cosa la sola nostra edizione della versione italiana potrà essere la prima in Italia, e andare di pari passo con la pubblicazione dell'originale. Gius. Vaccarino-Eredi Botta/Editori» (Gazzetta di Firenze, $\mathrm{n}^{\circ} 113$, giovedì 19 settembre $\left.1844, \mathrm{p} .4\right)$.

47. Citazione dalla lettera del 4 agosto, $431 / 23$.

48. D.M. Bruni, «La censura di Morfeo. Il controllo delle stampe nella Toscana della restaurazione", Clio, vol. 38, n 2, 2002, p. 203; A. De Rubertis, Studi sulla censura in Toscana, Pisa, Nistri-Lischi, 1936; Id., Nuovi studi sulla censura in Toscana con documenti inediti, Firenze, La Nuova Italia, 1951.

49. Si veda Le Siècle, $\mathrm{n}^{\circ} 210$, mardi 30 et mercredi 31 juillet 1844 ; Le Constitutionnel, $\mathrm{n}^{\circ} 213$, mercredi 31 juillet 1844 . 
luminara di Pisa per i bicchieri di vetro giallo, rosso, verde turchino e bianco, disposti nella simmetria più graziosa. La folla era grandissima dappertutto, ma il male avvenne all'imboccatura delle strade per l'intoppo delle persone che volevano uscire con quelle che volevano entrare, e ambedue difficilissime a contenersi anche dalla forza armata. Io la presi alla larga e me la passai benissimo $(431 / 23)$.

Con l'ironia che lo contraddistingue, il figlio Luigi postilla questo paragrafo con la formula «sussurri parigini ». Giuseppe Molini approfitta del basso volume di lavoro per una spedizione a Batignolles da Pietro Giannone, "così feci conoscenza con quel bravo vecchio che è l'autore dell'Esule», e per consegnargli una lettera del drammaturgo toscano Giovanni Battista Niccolini ${ }^{50}$. Sulla via del ritorno l'editore fiorentino fa visita al conte Terenzio Mamiani, a Parigi dal 1831 dopo i moti rivoluzionari e fra i protagonisti della cerchia di esuli che gravitava nella capitale francese ${ }^{51}$. Nella costruzione del dibattito risorgimentale gli inizi degli anni Quaranta sono nodali, sia per lo scontro, anche aspro, delle diverse idee di unità nazionale e su come ottenerla ${ }^{52}$, sia per un orizzonte sociale inedito ${ }^{53}$. Ci troviamo nel pieno di un percorso risorgimentale che a Parigi trae linfa vitale, sia per la cauta permissività della Monarchia di Luglio verso gli esuli della penisola, sia per la diffusione della cultura italiana nella capitale francese. L'epoca è «segnata dallo sviluppo esponenziale del mercato delle edizioni, dall'evoluzione significativa del ruolo e della funzione degli intellettuali e da un'immagine diversa dell'Italia animata da un fervido impulso patriottico-unitario e da una molteplicità di suggestioni europee ${ }^{54}$. Il rapporto transalpino è tutt'altro che unidirezionale: Baudry

50. Si veda la sua lettera del 27 giugno 1884, in Ricordi della vita e delle opere di G.-B. Niccolini, A. Vannucci (a cura di), Firenze, Le Monnier, vol. 2, 1866, p. 337.

51. «Nonostante fossero in continuo movimento, i membri della comunità degli esuli gravitavano attorno a un numero ristretto di centri intellettuali internazionali, la cui importanza trascendeva quella dei rispettivi paesi, e da essi traevano le loro idee. Parigi era indubbiamente il più importante di questi centri» (M. Isabella, Risorgimento in esilio. L'internazionale liberale e l'età delle rivoluzioni, Bari, Laterza, 2011, p. 39).

52. Giuseppe Mazzini considera Mamiani suo «ennemi acharné» a Parigi, insieme a venti altri; missiva di Mazzini a Thomas Émery da Londra il 21 luglio 1840, Lettres intimes de Joseph Mazzini, D. Melegari (a cura di), Parigi, Perrin, 1895, p. 230.

53. Tale da meritarsi l'appellativo di «decennio socialista». "Les années 1840 sont cruciales, les nombreuses tensions accumulées au cours des années précédentes s'intensifient. Le monde ouvrier est en ébullition, tout ce qui semblait oublié remonte à la surface» (M. Gribaudi, Paris ville ouvrière. Une histoire occultée (1789-1848), Parigi, La Découverte - Centre national du Livre, 2014, p. 333).

54. S. Tatti, «Bohème letteraria italiana a Parigi all'inizio dell'Ottocento», in Italia Italie. Immagini tra Rivoluzione e Restaurazione, S. Tatti (a cura di), Roma, Bulzoni, 1999, p. 160. 
e Galignani traggono ampi benefici dall'importazione e traduzione delle opere italiane. La sintesi editoriale che si configura ha "un effet non négligeable sur l'identité nationale et sur la culture aussi bien des exilés italiens que sur celle de leurs interlocuteurs ${ }^{55}$.

\section{Conclusioni}

Con il graduale esaurirsi delle commissioni, la liquidazione dei conti con i librai parigini ${ }^{56}$, in attesa di una risposta del ministro Paver che stenta ad arrivare, e con il proposito di tornare nella penisola, Giuseppe Molini si impegna in un dialogo intimo con Luigi Molini. Le lettere datate 11 e 13 agosto (431/24-25) convergono nel dare l'impressione che padre e figlio abbiano idee radicalmente diverse sulla conduzione della libreria-casa editrice. L'ironia nelle postille rosse di Luigi Molini ${ }^{57}$ cela un lungo pregresso, forse risalente agli anni del dissesto finanziario della ditta famigliare e al ritorno di Giuseppe Molini nella gestione, lasciata e ripresa negli anni della nomina a bibliotecario palatino. La lettera del 13 agosto è un terreno di scontro in absentia e uno squarcio nel pensiero di Luigi Molini. Non si tratta di risposte convenzionali, quali sarebbero se affidate a una lettera di risposta, bensì una riflessione con se stesso e il rimasticare pensieri interiori. L'impegno di Giuseppe Molini con il nuovo corrispondente Hachette evita secondo lui una commissione del $5 \%$ presso Duprat, ma va contro le precise disposizioni del figlio, che intende limitare al massimo gli impegni con altri editori stranieri ; allo stesso modo Luigi Molini non è d'accordo con l'idea del padre sui viaggiatori che presentano cataloghi, e che "al $99 \%$ puoi essere sicuro che ti mettono in mezzo ». Molini figlio non risparmia alcuni sprazzi di derisione quando appunta a lato dei paragrafi «Nonsense» e «Il sig. Giuseppe Molini non è contento neppure quando si fanno le cose in regola». La polemica si infiamma maggiormente quando gli argomenti sono i creditori, le scadenze sui pagamenti e le politiche scontistiche. Nella riflessione di Giuseppe Molini vi sono alcuni passaggi focali :

55. L. Fournier-Finocchiaro, «Les exilés politiques italiens, vecteurs et médiateurs de la langue et de la littérature italienne en France au XIX ${ }^{e}$ siècle», in Les exilés politiques espagnols, italiens et portugais en France au XIX ${ }^{e}$ siècle. Questions et perspectives, L. Fournier-Finocchiaro e C. Clímaco (a cura di), Parigi, L’Harmattan, 2017, p. 142.

56. Duprat, Klincksieck, Furne, Curmer, Guillaumin, Delalande, Brockhaus, solo per citarne alcuni.

57. «Solite sue gentilezze» in commento all'affermazione del padre «tu vuoi che si rispettino i tuoi sistemi ed io non dico nulla. Mi basta solo di guardarmi bene dall'imitarli », lettera datata 11 agosto, $431 / 24$. 
Voglio farti una osservazione e ti prego di credere sulla mia parola d'onore che quel che dico è con l'animo il più tranquillo del mondo, anzi con il sigaro in bocca e per il desiderio di vedere prosperare codesto negozio la di cui fondazione costò tanti sudori e stenti al mio ottimo padre, e il cui proseguimento è costato a me tante pene e poi tanti sacrifici ${ }^{58}[\ldots]$. Ricordati per l'amor di Dio che questi maledetti sconti rovinarono la casa Molini-Landi ${ }^{59}$ poi quella Veroli e poi il Masi, il Giachetti e altri mille. Del resto fai quello che credi ma sappi che gli imbarazzi nei quali ti trovi dipendono perché non hai voluto mai consultarmi prima di concludere degli affari viscosi. [...] E ho finito il discorso insieme con il sigaro (431/25).

Si tratta di nozioni essenziali per la raffigurazione del percorso della libreria-casa editrice Molini, che sarebbero rimaste sommerse senza l'indagine su questo carteggio. Allo stesso modo, gli squarci delle attività congiunte tra editori italiani e francesi contribuiscono ad allargare lo scenario culturale transalpino. Ne emerge un quadro ampio e sfaccettato, attraversato da impulsi risorgimentali e fermenti commerciali. Lo scopo dell'analisi è stato molteplice: offrire un panorama inedito nell'anno 1844 (a firma di un autorevole e navigato osservatore, addentro al sistema editoriale, quale era Giuseppe Molini), mettere a disposizione nuovi elementi intorno al commercio librario transalpino, e infine presentare un episodio significativo per la ricostruzione delle attività dei Molini, protagonisti del mondo del libro a Firenze e attenti a un orizzonte che travalicava le Alpi.

Marius Rusu

Università di Roma La Sapienza

58. La nota in rosso del figlio è impietosa: «Se vi fosse stato affezionato come dice non lo avrebbe prostituito nel 1828 con il metterci il nome Veroli». Luigi Molini fa riferimento al periodo in cui alla casa editrice della famiglia Molini, chiamata "All'insegna di Dante", subentrò l'editore Giuseppe Veroli di Bologna, si veda il Nuovo Giornale de' Letterati, Pisa, presso Sebastiano Nistri, t. 17, 1828, p. 207.

59. Il triplice sodalizio con Giuseppe Landi e Giovanni Rosini sotto l'intestazione «Molini, Landi, e C.» dura dal 1804 fino al 1815, anno in cui l'iniziativa comincia a sfaldarsi; si vedano le lettere di Giuseppe Molini a Giambattista Venturi conservate alla Biblioteca Panizzi di Reggio Emilia, Mss. A 27/26-22 e A 27/24-3. 